\title{
Existence of multiple solutions for the Brezis-Nirenberg-type problem with singular coefficients
}

Yang Yang ${ }^{1 *}$, Jihui Zhang ${ }^{2}$ and Xiusong $\mathrm{Gu}^{3}$

"Correspondence: yynjnu@126.com 'School of Science, Jiangnan University, Wuxi, 214122, China Full list of author information is available at the end of the article

\begin{abstract}
By energy estimates and by establishing a local (PS) condition, we obtain the multiplicity of solutions to a class of Brezis-Nirenberg-type problem with singular coefficients via minimax methods and the Krasnoselskii genus theory.
\end{abstract}

Keywords: Brezis-Nirenberg-type problem; minimax method

\section{Introduction and main results}

This paper is concerned with multiple solutions for the semilinear Brezis-Nirenberg-type problem with singular coefficients

$$
\begin{cases}-\operatorname{div}\left(\frac{D u}{|x|^{2 a}}\right)=\lambda \frac{|u|^{2 *-2} u}{|x|^{2 * b}}+\beta \frac{|u|^{q-2} u}{|x|^{\alpha}}, & x \in \Omega ; \\ u=0, & x \in \partial \Omega,\end{cases}
$$

where $\Omega \subset R^{n}$ is a bounded smooth domain, and $0 \in \Omega,-\infty<a<\frac{n-2}{2}, a \leq b<a+1$, $2^{*}=\frac{2 n}{n-2 d}, d=a+1-b \in(0,1], 1<q<2, \alpha<(1+a) q+n\left(1-\frac{q}{2}\right) . \beta>0, \lambda>0$ are two real parameters.

The starting point of the variational approach to the problem is the Caffarelli-KohnNirenberg inequality (see [1]): There is a constant $C_{a, b}>0$ such that

$$
\left(\int_{R^{n}}|x|^{-2 * b}|u|^{2 *} d x\right)^{2 / 2 *} \leq C_{a, b} \int_{R^{n}}|x|^{-2 a}|D u|^{2} d x
$$

for all $u \in C_{0}^{\infty}\left(R^{n}\right)$, where

$$
-\infty<a<\frac{n-2}{2}, \quad a \leq b<a+1, \quad 2 *=\frac{2 n}{n-2 d}, \quad d=a+1-b .
$$

Let $D_{a}^{1,2}(\Omega)$ be the completion of $C_{0}^{\infty}\left(R^{n}\right)$ with respect to the weighted norm $\|\cdot\|$ defined by

$$
\|u\|=\left(\int_{\Omega}|x|^{-2 a}|D u|^{2} d x\right)^{1 / 2} .
$$

(c) 2012 Yang et al.; licensee Springer. This is an Open Access article distributed under the terms of the Creative Commons Attribution License (http://creativecommons.org/licenses/by/2.0), which permits unrestricted use, distribution, and reproduction in any medium, provided the original work is properly cited. 
From the boundedness of $\Omega$ and the standard approximation arguments, it is easy to see that (2) holds for any $u \in D_{a}^{1,2}(\Omega)$ in the sense:

$$
\left(\int_{\Omega}|x|^{-\alpha}|u|^{r} d x\right)^{2 / r} \leq C \int_{\Omega}|x|^{-2 a}|D u|^{2} d x
$$

for $1 \leq r \leq 2^{*}=\frac{2 n}{n-2}, \frac{\alpha}{r} \leq(1+a)+n\left(\frac{1}{r}-\frac{1}{2}\right)$, that is, the embedding $D_{a}^{1,2}(\Omega) \hookrightarrow L^{r}\left(\Omega,|x|^{-\alpha}\right)$ is continuous, where $L^{r}\left(\Omega,|x|^{-\alpha}\right)$ is the weighted $L^{r}$ space with the norm

$$
\|u\|_{r, \alpha}:=\|u\|_{L^{r}\left(\Omega,|x|^{-\alpha}\right)}=\left(\int_{\Omega}|x|^{-\alpha}|u|^{r} d x\right)^{1 / r} .
$$

On $D_{a}^{1,2}(\Omega)$, we can define the energy functional

$$
J(u)=\frac{1}{2} \int_{\Omega}|x|^{-2 a}|D u|^{2} d x-\frac{\lambda}{2 *} \int_{\Omega}|x|^{-2 * b}|u|^{2 *} d x-\frac{\beta}{q} \int_{\Omega}|x|^{-\alpha}|u|^{q} d x .
$$

From (4), $J$ is well defined in $D_{a}^{1,2}(\Omega)$, and $J \in C^{1}\left(D_{a}^{1,2}(\Omega), R\right)$. Furthermore, the critical points of $J$ are weak solutions of problem (1).

Breiz-Nirenberg-type problems have been generalized to many situations such as

$$
\begin{cases}-\operatorname{div}\left(\frac{D u}{|x|^{2 a}}\right)-\mu \frac{u}{|x|^{2(a+1)}}=\frac{|u|^{2 *-2} u}{|x|^{2 s b}}+\lambda \frac{u}{|x|^{2(a+1)-c},} & x \in \Omega, \\ u=0, & x \in \partial \Omega .\end{cases}
$$

Xuan et al. [2] derived the explicit formula for the extremal functions of the best embedding constant by applying the Bliss lemma [3]. They got a nontrivial solution for problem (5) including the resonant and nonresonant cases by variational methods. He and Zou [4] studied problem (5) and obtained the multiplicity of solutions with the aid of a pseudoindex theory. In [5], problem (5) has been extended to the $p$-Laplace case by Xuan.

The purpose of this paper is to study the multiplicity of solutions for the BreizNirenberg-type problem (1) with the aid of a minimax method. We obtain multiple nontrivial solutions of (1) by proving the local (PS) condition and energy estimates.

Our main results are the following.

Theorem 1.1 Suppose $1<q<2$, then

(i) $\forall \beta>0, \exists \lambda_{0}>0$ such that if $0<\lambda<\lambda_{0}$, problem (1) has a sequence of solutions $\left\{u_{m}\right\}$ with $J\left(u_{m}\right)<0$ and $J\left(u_{m}\right) \rightarrow 0$ as $m \rightarrow \infty$.

(ii) $\forall \lambda>0, \exists \beta_{0}>0$ such that if $0<\beta<\beta_{0}$, problem (1) has a sequence of solutions $\left\{u_{m}\right\}$ with $J\left(u_{m}\right)<0$ and $J\left(u_{m}\right) \rightarrow 0$ as $m \rightarrow \infty$.

\section{Preliminary results}

Lemma 2.1 [5] Suppose that $\Omega \subset R^{n}$ is an open bounded domain with $C^{1}$ boundary and $0 \in \Omega,-\infty<a<(n-2) / 2$. The embedding $D_{a}^{1,2}(\Omega) \hookrightarrow L^{r}\left(\Omega,|x|^{-\alpha}\right)$ is compact if $1 \leq r<2$ ", $\alpha<(1+a) r+n\left(1-\frac{r}{2}\right)$.

Lemma 2.2 (Concentration compactness principle [5]) Let $-\infty<a<(n-2) / 2, a \leq b \leq$ $a+1,2 *=2 n /(n-2 d), d=1+a-b \in[0,1]$, and $M\left(R^{n}\right)$ be the space of bounded measures 
on $R^{n}$. Suppose that $\left\{u_{m}\right\} \subset D_{a}^{1,2}\left(R^{n}\right)$ is a sequence such that

$$
\begin{aligned}
& u_{m} \rightarrow u \quad \text { in } D_{a}^{1,2}\left(R^{n}\right), \\
& \mu_{m}:=\left.\left.|| x\right|^{-a} D u_{m}\right|^{2} d x \rightarrow \mu \quad \text { in } M\left(R^{n}\right), \\
& v_{m}:=\left.\left.|| x\right|^{-b} u_{m}\right|^{2 *} d x \rightarrow v \quad \text { in } M\left(R^{n}\right), \\
& u_{m} \rightarrow u \quad \text { a.e. on } R^{n} .
\end{aligned}
$$

Then there are the following statements:

(1) There exists some at most countable set $I$, a family $\left\{x^{(i)}: i \in I\right\}$ of distinct points in $R^{n}$, and a family $\left\{v^{(i)}: i \in I\right\}$ of positive numbers such that

$$
v=\left.\left.|| x\right|^{-b} u\right|^{2 *} d x+\sum_{i \in I} v^{(i)} \delta_{x^{(i)}},
$$

where $\delta_{x}$ is the Dirac-mass of mass 1 concentrated at $x \in R^{n}$.

(2) The following inequality holds

$$
\mu \geq\left.\left.|| x\right|^{-a} D u\right|^{2} d x+\sum_{i \in I} \mu^{(i)} \delta_{x^{(i)}}
$$

for some family $\left\{\mu^{(i)}>0: i \in I\right\}$ satisfying

$$
S\left(v^{(i)}\right)^{2 / 2^{*}} \leq \mu^{(i)} \quad \text { for all } i \in I,
$$

where $S:=\inf _{u \in D_{a}^{1,2}\left(R^{n}\right) \backslash\{0\}} E_{a, b}(u)$ to be the best embedding constants, and

$$
E_{a, b}(u)=\frac{\int_{R^{n}}|x|^{-2 a}|D u|^{2} d x}{\left(\int_{R^{n}}|x|^{-2 * b}|u|^{2 *} d x\right)^{2 / 2 *}} .
$$

In particular, $\sum_{i \in I}\left(v^{(i)}\right)^{2 / 2 *}<\infty$.

Lemma 2.3 Assume $\left\{u_{n}\right\}$ is a $(P S)_{c}$ sequence with $c<0,1<q<2$, then

(1) $\forall \lambda>0$, there exists $\beta_{1}>0$ such that for any $0<\beta<\beta_{1},\left\{u_{n}\right\}$ has a convergent subsequence in $D_{a}^{1,2}(\Omega)$.

(2) $\forall \beta>0$, there exists $\lambda_{1}>0$ such that for any $0<\lambda<\lambda_{1},\left\{u_{n}\right\}$ has a convergent subsequence in $D_{a}^{1,2}(\Omega)$.

Proof (1) The boundedness of (PS) ${ }_{c}$ sequence.

For $\left\{u_{n}\right\}$ is a (PS) $)_{c}$ sequence, then

$$
\begin{aligned}
& J\left(u_{n}\right)=\frac{1}{2} \int_{\Omega}|x|^{-2 a}\left|D u_{n}\right|^{2} d x-\frac{\lambda}{2 *} \int_{\Omega}|x|^{-2 * b}\left|u_{n}\right|^{2 *} d x-\frac{\beta}{q} \int_{\Omega}|x|^{-\alpha}\left|u_{n}\right|^{q} d x, \\
& \left\langle J^{\prime}\left(u_{n}\right), u_{n}\right\rangle=\int_{\Omega}|x|^{-2 a}\left|D u_{n}\right|^{2} d x-\lambda \int_{\Omega}|x|^{-2 * b}\left|u_{n}\right|^{2 *} d x-\beta \int_{\Omega}|x|^{-\alpha}\left|u_{n}\right|^{q} d x .
\end{aligned}
$$


So, we get

$$
\begin{aligned}
o(1)\left(1+\left\|u_{n}\right\|\right)+|c| & \geq J\left(u_{n}\right)-\frac{1}{2 *}\left\langle J^{\prime}\left(u_{n}\right), u_{n}\right\rangle \\
& =\frac{d}{n}\left\|u_{n}\right\|^{2}-\left(\frac{1}{q}-\frac{1}{2 *}\right) \beta \int_{\Omega} \frac{\left|u_{n}\right|^{q}}{|x|^{\alpha}} d x \\
& \geq \frac{d}{n}\left\|u_{n}\right\|^{2}-\left(\frac{1}{q}-\frac{1}{2 *}\right) \beta C_{\alpha}\left\|u_{n}\right\|^{q} .
\end{aligned}
$$

We have the boundedness of $\left\{u_{n}\right\}$ for $1<q<2$, then there exists a subsequence, we still denote it by $\left\{u_{n}\right\}$, such that

$$
\begin{aligned}
& u_{n} \rightarrow u \quad \text { in } D_{a}^{1,2}(\Omega), \\
& u_{n} \rightarrow u \quad \text { in } L^{2 *}\left(\Omega,|x|^{-2 * b}\right), \\
& u_{n} \rightarrow u \quad \text { in } L^{r}\left(\Omega,|x|^{-\alpha}\right), \forall 1 \leq r<\frac{2 n}{n-2}, \alpha<(1+a) r+n\left(1-\frac{r}{2}\right), \\
& u_{n} \rightarrow u \quad \text { a.e. on } \Omega .
\end{aligned}
$$

From the concentration compactness principle, there exist nonnegative measures $\mu, v$ and a countable family $\left\{x_{i}\right\} \subset \Omega$ such that

$$
\begin{aligned}
& |x|^{-2 \cdot b}\left|u_{n}\right|^{2 a} d x-v=|x|^{-2 \cdot b}|u|^{2 a} d x+\sum_{i \in I} v^{(i)} \delta_{x^{(i)}}, \\
& |x|^{-2 a}\left|D u_{n}\right|^{2} d x \rightarrow \mu \geq|x|^{-2 a}|D u|^{2} d x+S \sum_{i \in I}\left(v^{(i)}\right)^{2 / 2 *} \delta_{x^{(i)}} .
\end{aligned}
$$

(2) Up to a subsequence, $u_{n} \rightarrow u$ in $L^{2 *}\left(\Omega,|x|^{-2 * b}\right)$.

Since $\left\{u_{n}\right\}$ is bounded in $D_{a}^{1,2}(\Omega)$, we may suppose, without loss of generality, that there exists $T \in\left(L^{2^{\prime}}\left(\Omega,|x|^{-2 a}\right)\right)^{n}$ such that

$$
D u_{n} \rightarrow T \quad \text { in }\left(L^{2^{\prime}}\left(\Omega,|x|^{-2 a}\right)\right)^{n} .
$$

On the other hand, $\left|u_{n}\right|^{2 *-2} u_{n}$ is also bounded in $L^{2_{s}^{\prime}}\left(\Omega,|x|^{-2 * b}\right)$ and

$$
\left|u_{n}\right|^{2 *-2} u_{n} \rightarrow|u|^{2 *-2} u \quad \text { in } L^{2{ }^{\prime}}\left(\Omega,|x|^{-2 * b}\right) .
$$

Note that

$$
\begin{aligned}
o(1)\|\varphi\|= & \left\langle J^{\prime}\left(u_{n}\right), \varphi\right\rangle \\
= & \int_{\Omega}|x|^{-2 a} D u_{n} D \varphi d x-\lambda \int_{\Omega}|x|^{-2 \cdot b}\left|u_{n}\right|^{2 \cdot-2} u_{n} \varphi d x \\
& -\beta \int_{\Omega}|x|^{-\alpha}\left|u_{n}\right|^{q-2} u_{n} \varphi d x,
\end{aligned}
$$

taking $n \rightarrow \infty$ in (11), we have

$$
\int_{\Omega}|x|^{-2 a} T D \varphi d x=\lambda \int_{\Omega}|x|^{-2 * b}|u|^{2 s-2} u \varphi d x+\beta \int_{\Omega}|x|^{-\alpha}|u|^{q-2} u \varphi d x
$$


for any $\varphi \in D_{a}^{1,2}(\Omega)$. Let $\varphi=\psi u_{n}$ in (12), where $\psi \in C(\bar{\Omega})$, then it follows that

$$
\begin{aligned}
& \int_{\Omega}|x|^{-2 a} D u_{n} u_{n} D \psi d x+\int_{\Omega}|x|^{-2 a}\left|D u_{n}\right|^{2} \psi d x \\
& =\lambda \int_{\Omega}|x|^{-2 * b}\left|u_{n}\right|^{2 *} \psi d x+\beta \int_{\Omega}|x|^{-\alpha}\left|u_{n}\right|^{q} \psi d x .
\end{aligned}
$$

Taking $n \rightarrow \infty$ in (13), we have

$$
\int_{\Omega}|x|^{-2 a} u T D \psi d x+\int_{\Omega} \psi d \mu=\lambda \int_{\Omega} \psi d v+\beta \int_{\Omega}|x|^{-\alpha}|u|^{q} \psi d x
$$

Let $\varphi=\psi u$ in (12), then it follows that

$$
\int_{\Omega}|x|^{-2 a} T \psi u d x+\int_{\Omega}|x|^{-2 a} T u d \psi=\lambda \int_{\Omega}|x|^{-2 * b}|u|^{2^{*}} \psi d x+\beta \int_{\Omega}|x|^{-\alpha}|u|^{q} \psi d x .
$$

Thus, it implies that

$$
\int_{\Omega} \psi d \mu=\lambda \sum_{i \in I} v_{i} \psi\left(x_{i}\right)+\int_{\Omega}|x|^{-2 a} T D u \psi d x
$$

which implies that

$$
S\left(v_{i}\right)^{2 / 2 "} \leq \mu_{i}=\lambda v_{i}
$$

Hence, $v_{i} \geq\left(\lambda^{-1} S\right)^{n / 2 d}$ if $v_{i} \neq 0$.

On the other hand,

$$
\begin{aligned}
0 & >c=\lim _{n \rightarrow \infty}\left(J\left(u_{n}\right)-\frac{1}{2 *}\left\langle J^{\prime}\left(u_{n}\right), u_{n}\right\rangle\right) \\
& =\lim _{n \rightarrow \infty}\left(\frac{d}{n}\left\|u_{n}\right\|^{2}-\beta\left(\frac{1}{q}-\frac{1}{2 *}\right) \int_{\Omega}|x|^{-\alpha}\left|u_{n}\right|^{q} d x\right) \\
& \geq \frac{d}{n}\|u\|^{2}-\beta C\|u\|^{q},
\end{aligned}
$$

then $\|u\|^{q} \leq C \beta^{q /(2-q)}$, so that

$$
\begin{aligned}
0 & >c=\lim _{n \rightarrow \infty}\left(J\left(u_{n}\right)-\frac{1}{2 *}\left\langle J^{\prime}\left(u_{n}\right), u_{n}\right\rangle\right) \\
& =\lim _{n \rightarrow \infty}\left(\frac{d}{n}\left\|u_{n}\right\|^{2}-\beta\left(\frac{1}{q}-\frac{1}{2 *}\right) \int_{\Omega}|x|^{-\alpha}\left|u_{n}\right|^{q} d x\right) \\
& \geq \frac{d}{n} \mu_{i}-\beta C \beta^{q /(2-q)} \\
& \geq \frac{d}{n} S^{\frac{n}{2 d}}\left(\lambda^{-1}\right)^{\frac{n-2 d}{2 d}}-C \beta^{\frac{2}{2-q}}
\end{aligned}
$$

However, if $\beta>0$ is given, we can choose $\lambda_{1}>0$ so small that for every $0<\lambda<\lambda_{1}$, the last term on the right-hand side above is greater than 0 , which is a contradiction. Similarly, if 
$\lambda>0$ is given, we can take $\beta_{1}>0$ so small that for every $0<\beta<\beta_{1}$, the last term on the right-hand side above is greater than 0 . Then $v_{i}=0$ for each $i$.

Up to now, we have shown that

$$
\lim _{n \rightarrow \infty} \int_{\Omega}|x|^{-2 * b}\left|u_{n}\right|^{2 *} d x=\int_{\Omega}|x|^{-2 * b}|u|^{2 *} d x
$$

So, by the Breiz-Lieb lemma,

$$
\begin{aligned}
o(1)\left\|u_{n}\right\| & =\left\|u_{n}\right\|^{2}-\lambda \int_{\Omega}|x|^{-2 \cdot b}\left|u_{n}\right|^{2 \cdot} d x-\beta \int_{\Omega}|x|^{-\alpha}\left|u_{n}\right|^{q} d x \\
& =\left\|u_{n}-u\right\|^{2}-\|u\|^{2}-\lambda \int_{\Omega}|x|^{-2 * b}|u|^{2 *} d x-\beta \int_{\Omega}|x|^{-\alpha}|u|^{q} d x \\
& =\left\|u_{n}-u\right\|^{2}+o(1)\|u\|
\end{aligned}
$$

since $J^{\prime}(u)=0$. Thus, we prove that $\left\{u_{n}\right\}$ strongly converges to $u$ in $D_{a}^{1,2}(\Omega)$.

\section{Existence of infinitely many solutions}

In this section, we use the minimax procedure to prove the existence of infinitely many solutions. Let $\Sigma$ be the class of subsets of $D_{a}^{1,2}(\Omega) \backslash\{0\}$, which are closed and symmetric with respect to the origin. For $A \in \Sigma$, we define the genus $\gamma(A)$ by

$$
\gamma(A)=\min \left\{k \in N: \exists \phi \in C\left(A, R^{k} \backslash\{0\}\right), \phi(x)=-\phi(-x)\right\} .
$$

Assume that $1<q<2$, then we obtain

$$
\begin{aligned}
J(u) & =\frac{1}{2} \int_{\Omega}|x|^{-2 a}|D u|^{2} d x-\frac{\lambda}{2 *} \int_{\Omega}|x|^{-2 * b}|u|^{2 *} d x-\frac{\lambda}{q} \int_{\Omega}|x|^{-\alpha}|u|^{q} d x \\
& \geq \frac{1}{2}\|u\|^{2}-\frac{C_{b} \lambda}{2 *}\|u\|^{2 *}-\frac{\beta C_{\alpha}}{q}\|u\|^{q} .
\end{aligned}
$$

Define

$$
h(t)=\frac{1}{2} t^{2}-\lambda C_{1} t^{2 *}-\beta C_{2} t^{q} .
$$

Then, given $\beta>0$, there exists $\lambda_{2}>0$ so small that for every $0<\lambda<\lambda_{2}$, there exists $0<T_{0}<$ $T_{1}$ such that $h(t)<0$ for $0<t<T_{0}, h(t)>0$ for $T_{0}<t<T_{1}, h(t)<0$ for $t>T_{1}$. Similarly, given $\lambda>0$, we can choose $\beta_{2}>0$ with the property that $T_{0}, T_{1}$ as above exist for each $0<\beta<\beta_{2}$. Clearly, $h\left(T_{0}\right)=h\left(T_{1}\right)=0$. Following the same idea as in [6-8], we consider the truncated functional

$$
\tilde{J}(u)=\frac{1}{2} \int_{\Omega}|x|^{-2 a}|D u|^{2} d x-\frac{\lambda}{2 *} \psi(u) \int_{\Omega}|x|^{-2 * b}|u|^{2 *} d x-\frac{\lambda}{q} \int_{\Omega}|x|^{-\alpha}|u|^{q} d x,
$$

where $\psi(u)=\tau(\|u\|)$, and $\tau: R^{+} \rightarrow[0,1]$ is a nonincreasing $C^{\infty}$ function such that $\tau(t)=1$ if $t \leq T_{0}$ and $\tau(t)=0$ if $t \geq T_{1}$. The main properties of $\tilde{J}$ are the following. 


\section{Lemma 3.1}

(1) $\tilde{J} \in C^{1}$ and $\tilde{J}$ is bounded below.

(2) If $\tilde{J}(u) \leq 0$, then $\|u\| \leq T_{0}$ and $\tilde{J}(u)=J(u)$.

(3) For any $\lambda>0$, there exists $\beta_{0}=\min \left\{\beta_{1}, \beta_{2}\right\}$ such that if $0<\beta<\beta_{0}$ and $c<0$, then $\tilde{J}$ satisfies $(P S)_{c}$ condition.

(4) for any $\beta>0$, there exists $\lambda_{0}=\min \left\{\lambda_{1}, \lambda_{2}\right\}$ such that if $0<\lambda<\lambda_{0}$ and $c<0$, then $\tilde{J}$ satisfies $(P S)_{c}$ condition.

Proof (1) and (2) are immediate. To prove (3) and (4), observe that all (PS) ${ }_{c}$ sequences for $\tilde{J}$ with $c<0$ must be bounded. Similar to the proof of Lemma 2.3, there exists a convergent subsequence.

Lemma 3.2 Given $m \in N$, there is $\varepsilon_{m}<0$ such that

$$
\gamma\left(\left\{u \in D_{a}^{1,2}(\Omega): \tilde{J}(u) \leq \varepsilon_{m}\right\}\right) \geq m .
$$

Proof Fix $m$ and let $H_{m}$ be an $m$-dimensional subspace of $D_{a}^{1,2}(\Omega)$. Take $u \in H_{m}, u \neq 0$, write $u=r_{m} v$ with $v \in H_{m},\|v\|=1$ and $r_{m}=\|u\|$. Thus, for $0<r_{m}<T_{0}$, since all the norms are equivalent, we have

$$
\begin{aligned}
\tilde{J}(u) & =J(u)=\frac{1}{2} \int_{\Omega}|x|^{-2 a}|D u|^{2} d x-\frac{\lambda}{2 *} \int_{\Omega}|x|^{-2 * b}|u|^{2 *} d x-\frac{\lambda}{q} \int_{\Omega}|x|^{-\alpha}|u|^{q} d x \\
& \leq \frac{1}{2}\|u\|^{2}-\frac{\lambda C_{1}}{2 *}\|u\|^{2 *}-\frac{\lambda C_{2}}{q}\|u\|^{q} \\
& =\frac{1}{2} r_{m}^{2}-\frac{\lambda C_{1}}{2 *} r_{m}^{2 *}-\frac{\lambda C_{2}}{q} r_{m}^{q}:=\varepsilon_{m} .
\end{aligned}
$$

Therefore, we can choose $r_{m} \in\left(0, T_{0}\right)$ so small that $\tilde{J}(u) \leq \varepsilon_{m}<0$. Let $S_{r_{m}}=\left\{u \in D_{a}^{1,2}(\Omega)\right.$ : $\left.\|u\|=r_{m}\right\}$, then $S_{r_{m}} \cap H_{m} \subset \tilde{J}^{\varepsilon_{m}}$. Hence, $\gamma\left(\tilde{J}^{\varepsilon m}\right) \geq \gamma\left(S_{r_{m}} \cap H_{m}\right)=m$. Denote $\Gamma_{m}=\{A \in \Sigma$ : $\gamma(A) \geq m\}$ and let

$$
c_{m}=\inf _{A \in \Gamma_{m}} \sup _{u \in A} \tilde{J}(u) .
$$

Then $-\infty<c_{m} \leq \varepsilon_{m}<0$ because $\tilde{J}^{\varepsilon_{m}} \in \Gamma_{m}$ and $\tilde{J}$ is bounded from below.

Lemma 3.3 Let $\lambda, \beta$ be as in (3) or (4) of Lemma 3.1. Then all $c_{m}$ are critical values of $\tilde{J}$ as $c_{m} \rightarrow 0$.

Proof It is clear that $c_{m} \leq c_{m+1}, c_{m}<0$. Hence, $c_{m} \rightarrow \bar{c} \leq 0$. Moreover, since all $c_{m}$ are critical values of $\tilde{J}$, we claim that $\bar{c}=0$. If $\bar{c}<0$, because $K_{\bar{c}}$ is compact and $K_{\bar{c}} \in \Sigma$, it follows that $\gamma\left(K_{\bar{c}}\right)=N_{0}<+\infty$ and there exists $\delta>0$ such that $\gamma\left(K_{\bar{c}}\right)=\gamma\left(N_{\delta}\left(K_{\bar{c}}\right)\right)=N_{0}$. By the deformation lemma there exist $\varepsilon>0(\bar{c}+\varepsilon<0)$ and an odd homeomorphism $\eta$ such that

$$
\eta\left(\tilde{J}^{\bar{c}+\varepsilon} \backslash N_{\delta}\left(K_{\bar{c}}\right)\right) \subset \tilde{J}^{\bar{c}-\varepsilon} .
$$


Since $c_{m}$ is increasing and converges to $\bar{c}$, there exists $m \in N$ such that $c_{m}>\bar{c}-\varepsilon$ and $c_{m+N_{0}} \leq \bar{c}$ and there exists $A \in \Gamma_{m+N_{0}}$ such that $\sup _{u \in A} \tilde{J}(u)<\bar{c}+\varepsilon$. By the properties of $\gamma$, we have

$$
\gamma\left(\overline{A \backslash N_{\delta}\left(K_{\bar{c}}\right)}\right) \geq \gamma(A)-\gamma\left(N_{\delta}\left(K_{\bar{c}}\right)\right) \geq m, \quad \gamma\left(\overline{A \backslash N_{\delta}\left(K_{\bar{c}}\right)}\right) \geq m
$$

Therefore,

$$
\eta\left(\overline{A \backslash N_{\delta}\left(K_{\bar{c}}\right)}\right) \in \Gamma_{m} .
$$

Consequently,

$$
\sup _{u \in \eta\left(\overline{\left.A \backslash N_{\delta}\left(K_{\bar{c}}\right)\right)}\right.} \tilde{J}(u) \geq c_{m}>\bar{c}-\varepsilon,
$$

a contradiction, hence $c_{m} \rightarrow 0$.

\section{With Lemma 3.1 to Lemma 3.3, we have proved Theorem 1.1.}

\section{Competing interests}

The authors declare that they have no competing interests.

\section{Authors' contributions}

All authors read and approved the final manuscript.

\section{Author details}

'School of Science, Jiangnan University, Wuxi, 214122, China. ${ }^{2}$ School of Mathematical Science, Nanjing Normal University, Nanjing, 210097, China. ${ }^{3}$ Institute of Science, PLA University of Science and Technology, Nanjing, 211101, China.

\section{Acknowledgements}

Project is supported by National Natural Science Foundation of China, Tian Yuan Special Foundation (No. 11226116), the China Scholarship Council, Natural Science Foundation of Jiangsu Province of China for Young Scholar (No. BK2012109), the Fundamental Research Funds for the Central Universities (No. JUSRP11118, JUSRP211A22) and Foundation for young teachers of Jiangnan University (No. 2008LQN008).

Received: 4 September 2012 Accepted: 19 September 2012 Published: 26 November 2012

\section{References}

1. Caffarelli, I, Kohn, R, Nirenberg, L: First order interpolation inequalities with weights. Compos. Math. 53, 259-275 (1984)

2. Xuan, B, Su, S, Yan, Y: Existence results for Brezis-Nirenberg problems with Hardy potential and singular coefficients. Nonlinear Anal. 67, 2091-2106 (2007)

3. Bliss, G: An integral inequality. J. Lond. Math. Soc. 5, 40-46 (1930)

4. He, XM, Zou, WM: Multiple solutions for the Brezis-Nirenberg problem with a Hardy potential and singular coefficients. Comput. Math. Appl. 56, 1025-1031 (2008)

5. Xuan, B: The solvability of quasilinear Brezis-Nirenberg-type problems with singular weights. Nonlinear Anal. 62, 703-725 (2005)

6. Bernis, F, Garcia-Azorero, J, Peral, I: Existence and multiplicity of nontrivial solutions in semilinear critical problems of fourth-order. Adv. Differ. Equ. 1, $219-240$ (1996)

7. Wang, YJ, Shen, YT: Multiple and sign-changing solutions for a class of semilinear biharmonic equation. J. Differ. Equ. 246, 3109-3125 (2009)

8. Wang, YJ, Yin, YM, Yao, YT: Multiple solutions for quasilinear Schrödinger equations involving critical exponent. Appl. Math. Comput. 216, 849-856 (2010)

doi:10.1186/1687-2770-2012-137

Cite this article as: Yang et al.: Existence of multiple solutions for the Brezis-Nirenberg-type problem with singular coefficients. Boundary Value Problems 2012 2012:137. 